\title{
Microvesicle Tissue Factor Activity and IL-8 Levels are Associated with Mortality in Patients with Influenza A/H1N1 Infection
}

\author{
Matthew T. Rondina, MD, MS, FAHA ${ }^{1,2,3}$, Kohei Tatsumi, MD ${ }^{4}$, Julie A. Bastarache, MD $^{5}$, and \\ Nigel Mackman, PhD, FAHA ${ }^{4}$ \\ ${ }^{1}$ Division of General Internal Medicine, University of Utah School of Medicine, Salt Lake City, \\ Utah, 84132 \\ ${ }^{2}$ Division of Molecular Medicine Program at the University of Utah School of Medicine, Salt Lake \\ City, Utah, 84132 \\ ${ }^{3}$ Department of Internal Medicine at the George E. Wahlen Salt Lake City VAMC \\ ${ }^{4}$ Division of Hematology/Oncology, Department of Medicine, UNC McAllister Heart Institute, \\ University of North Carolina at Chapel Hill, NC, 27599 \\ ${ }^{5}$ Division of Allergy, Pulmonary, and Critical Care Medicine, Vanderbilt University Medical Center, \\ Nashville, TN, 37232
}

\section{Abstract}

Objective-To identify plasma biomarkers that can be early predictors of mortality in critically ill patients with primary influenza A/H1N1.

Design-A prospective, multicenter, case-cohort pilot study.

Setting-Three academic ICUs.

Patients-Fifteen patients with primary influenza A/H1N1 that included 7 survivors and 8 nonsurvivors. For comparison, age- and gender-matched healthy controls $(n=27)$ were also studied.

Interventions-Plasma was prepared from whole blood drawn upon ICU admission in influenza patients (ICU day 1). Microvesicle tissue factor (MV-TF) activity, thrombin-antithrombin complexes (TATc), and D-dimers were measured as procoagulant markers and markers of activation of coagulation. Plasma cytokine levels were measured on the same blood samples in a subset of 12 influenza patients using the Luminex Multi-Analyte Profiling system. Patients were followed for the primary outcome of 28-day mortality.

Measurements and Main Results-The average admission APACHE II score of the patients was $25.5 \pm 9.3,60 \%$ of patients had shock, and the 28 -day mortality rate was $53.3 \%(n=8 / 15)$. Influenza patients had dysregulated indices of coagulation and inflammation compared to controls. Among the markers of activation of coagulation measured on ICU day 1, only increased MV-TF

Corresponding Author and Address for Reprints: Matthew T. Rondina, MD, Eccles Institute of Human Genetics, 15 North 2030 East, Room 4220, Salt Lake City, Utah 84112, (801) 585-0950 (phone), (801) 585-0701 (fax), matthew.rondina @ hsc.utah.edu. 
activity was significantly associated with subsequent influenza-related mortality $(5.6 \pm 1.2 \mathrm{pg} / \mathrm{ml}$ in non-survivors vs. $1.8 \pm 0.8 \mathrm{pg} / \mathrm{mL}$ in survivors, $\mathrm{p}<0.05$ ). IL- 8 was significantly higher in nonsurvivors compared with survivors $(71.8 \pm 29.1 \mathrm{pg} / \mathrm{ml}, \mathrm{n}=5$ vs. $17.3 \pm 3.7 \mathrm{pg} / \mathrm{mL}, \mathrm{n}=7, \mathrm{p}<0.05)$. In addition, MV-TF activity and IL-8 levels were significantly and positively correlated $\left(\mathrm{r}^{2}=0.60\right.$, $P=0.003)$. Other cytokines, TATc, and D-dimer were not different between non-survivors and survivors and did not correlate with illness severity or mortality.

Conclusions-This study identifies an association between plasma IL-8 and MV-TF activity measured upon admission in patients with severe, primary influenza $\mathrm{A} / \mathrm{H} 1 \mathrm{~N} 1$ infection and subsequent mortality. Thus, these biomarkers may serve as very early prognostic markers for patients with influenza A/H1N1.

\section{Keywords}

Influenza; Microvesicles; Tissue Factor; Interleukin-8; Mortality; Acute Respiratory Distress Syndrome; Viral Diseases

\section{Introduction}

Influenza $\mathrm{A} / \mathrm{H} 1 \mathrm{~N} 1$ is a single-stranded RNA virus that causes a high number of intensive care unit (ICU) admissions, as well as considerable morbidity and mortality. Although effective treatments for influenza exist, treatment and triage decisions are still difficult because of a lack of accurate, reliable severity of illness predictive scores to determine the need for ICU admission (1). Patients with influenza have alterations in hemostatic parameters, thrombocytopenia, disseminated intravascular coagulation (DIC), and thrombosis (2). We have shown that platelets are activated in critically ill patients with primary 2009 influenza A /H1N1 (3). In addition, influenza A/H1N1 infection causes a "cytokine storm". Given these prominent alterations in hemostasis and inflammation during influenza and their contribution to adverse outcomes, we sought to determine whether early indices of dysregulated coagulation and inflammation would be associated with influenzarelated mortality.

Circulating microvesicles (MVs, also called microparticles or extracellular vesicles) are small membrane fragments that can transfer mRNA and proteins between cells (4). MVs are particularly prothrombotic when displaying tissue factor (TF) (4). Viruses, such as influenza, induce TF expression in monocytes and endothelial cells and activate the coagulation cascade, which can result in DIC (5). Influenza virus H1N1 also activates platelets and induces the release of MVs (6). However, there are no studies reporting levels of circulating TF-expressing MVs during the course of human influenza. In this study, we measured levels of circulating MV-TF (MV-TF) activity and plasma cytokines in critically ill patients admitted with primary influenza $\mathrm{A} / \mathrm{H} 1 \mathrm{~N} 1$ and tested their association with adverse clinical outcomes. 


\section{Materials and Methods}

\section{Human Subjects Enrollment}

This was a prospective study of patients $(\mathrm{n}=15)$ with primary influenza $\mathrm{A} / \mathrm{H} 1 \mathrm{~N} 1$ aged $\geq 21$ years enrolled within 24 hours of ICU admission. For comparison, age- and gender-matched, healthy, medication free, non-hospitalized, consenting control subjects aged $\geq 21$ were also studied ( $\mathrm{n}=27$ ). The Institutional Review Boards approved this study (protocols 28210 and 1005443) and all subjects or a legally authorized representative provided written informed consent. Influenza was diagnosed by reverse transcription polymerase chain reaction on a respiratory sample (Roche Applied Science, Germany). Patients with concurrent, secondary bacterial infection upon admission were excluded. The development of bacterial infections during patients' ICU course was evaluated by blood, sputum, and urine cultures and serologies. Demographics, laboratory data, and illness severity scores were captured on all patients. Overt DIC was calculated according to the ISTH criteria, which has a high sensitivity and specificity for DIC but has some limitations such as accounting for temporal changes. Patients were followed prospectively for the primary outcome of all-cause, 28-day mortality.

\section{Measurement of MV-TF activity, Markers of Activation of Coagulation and Cytokines}

Plasma was prepared from ACD-anticoagulated whole blood drawn at the time of study enrollment via centrifugation (1500rpm for 20 minutes). Plasma was immediately frozen at $-80^{\circ} \mathrm{C}$ until MV-TF, coagulation, and cytokine measurements. The levels of MV-TF activity in plasma were measured by an in-house two-step FXa generation assay (7). Briefly, MVs are pelleted by centrifugation of $200 \mu \mathrm{L}$ of plasma and washed twice to remove the plasma. MVs are re-suspended in buffer and incubated with FVIIa (2.5 nM), FX (300 nM) and $\mathrm{CaCl}_{2}(10 \mathrm{mM})$ for 2 hours at $37^{\circ} \mathrm{C}$ in the presence of either an inhibitory anti-TF antibody (HTF-1) or a control antibody. Finally, levels of FXa are determined by adding a chromogenic substrate for 15 minutes at $37^{\circ} \mathrm{C}$ and measuring the absorbance at $405 \mathrm{nM}$ with a VERSAmax microplate reader. The level of MV-TF activity in healthy controls is $<0.05$ $\mathrm{pg} / \mathrm{mL}$. Recombinant human TF (Innovin; Dade Berhing, Marburg, Germany) was used as a standard. Plasma levels of thrombin antithrombin complexes (TATc, normal $<4.0 \mathrm{ng} / \mathrm{mL}$ ) and D-dimer (normal $<500 \mathrm{ng} / \mathrm{mL}$ ) were quantified by ELISA using the TAT Enzygnost Micro Kit (Dade Behring/Siemens) and Asserachrom D-dimer kit (Diagnostica Stago), respectively. Plasma cytokine levels were measured using the Luminex Multi-Analyte Profiling system (LabMAPTM) technology.

\section{Statistical Analyses}

Data were examined for normality using skewness and kurtosis tests. Summary statistics were determined using STATA (v11.0). Groups were compared using the Student's $t$-test and the Chi-squared test, as appropriate (GraphPad Software, v5.0; STATA v11.0). Regression analyses were used to identify significant correlations between coagulation parameters and mortality. A two-tailed $P$ value $<0.05$ was considered significant. 


\section{Results}

Upon ICU admission, all influenza A/H1N1 patients had acute respiratory distress requiring intubation and more than $50 \%$ of patients had shock requiring vasopressors (Table 1). Control subjects were well matched to influenza patient on the variables of age and gender, and as expected had very low levels of MV-TF activity (Table 1). Influenza patients had significantly higher plasma fibrinogen levels $(571 \pm 67 \mathrm{mg} / \mathrm{dL}$ vs. $308 \pm 18 \mathrm{mg} / \mathrm{dL}, P<0.001)$ and $\mathrm{C}$-reactive protein $(11.0 \pm 2$ vs. $2.2 \pm 0.9, P<0.001)$, consistent with a systemic inflammatory response and activation of the coagulation system during influenza.

However, while procoagulant markers and markers of activation of coagulation were all increased in patients with influenza compared to controls, only MV-TF activity was associated with influenza-related mortality, being significantly higher in non-survivors compared with survivors (Table 1, Figure 1A). Platelet counts and fibrinogen levels were slightly lower in non-survivors but did not reach significance (Table 1). MV-TF activity, TATc levels, and D-dimer did not correlate with APACHE II score, platelet count, fibrinogen levels, CRP, or age and did not significantly differ between male and female influenza patients or between patients with severe sepsis versus septic shock (not shown).

Next, we examined plasma cytokine levels in influenza patients and controls. IL-2R, IL-8 and IL-10 were increased in patients with influenza $A / H 1 N 1$ compared to control subjects whereas IL-1 $\beta$, IL-2, IL-4, IL-5. IL-12, IL-13, IL-17, and CD40L were not changed (Table 1). We previously reported that IL-6 is also increased in influenza A/H1N1 patients (3), consistent with the systemic inflammatory milieu during influenza. However, within the group of cytokines that increased in patients only IL-8 was significantly associated with influenza-related mortality, being higher in non-survivors compared with survivors (Figure 1B). Interestingly, MV-TF activity significantly correlated with IL-8 levels (Figure 1C).

\section{Discussion}

In this study, we report that plasma MV-TF activity and IL-8 levels are increased in patients with primary influenza A/H1N1 and are significantly associated with influenza-related mortality. Our study is consistent with a previous paper that showed four chemokines (IL-8, IP-10, MCP-1 and MIG) were associated with fatal outcome in patients infected with influenza A (H5N1)(8). Other procoagulant markers and markers of inflammation or activation of coagulation were generally increased in patients with influenza but did not differ between survivors and non-survivors. Influenza virus can induce the expression of $\mathrm{TF}$ and the release of MVs, promoting a dysregulated pro-coagulant milieu (6). We posit that MV-TF activity in patients with influenza may not merely be a marker of severe infectious disease but may also contribute to adverse events such as vascular thrombosis, organ failure, and death. Further studies are needed to extend these studies and to examine the biological functions of MV-TF during influenza infection.

Our measurements of circulating MV-TF activity and IL-8 levels were made within 24 hours of ICU admission and robustly identified patients who did not survive their infectious course. As such, MV-TF activity and IL-8 may have utility as an early prognostic marker for 
patients with influenza $\mathrm{A} / \mathrm{H} 1 \mathrm{~N} 1$. These findings build on and extend observations made in bacterial sepsis and acute allograft rejection, where TF+ MVs correlate with adverse clinical outcomes (9-11). Moreover, since early plasma-based biomarkers for predicting influenzarelated mortality remain largely absent, these results may be utilized to improve prognostication and risk-stratification efforts in the early stages of treatment for patients with severe influenza. In addition, our findings may help identify patients where more intensive monitoring or treatment may be warranted, for example admission to an ICU setting.

The strengths of this study include its prospective multi-center design, robust determination of illness severity and complications, comprehensive characterization of markers of systemic activation of coagulation and inflammation, and inclusion of a matched healthy control cohort. The primary limitation of the study is the small sample size and therefore one must be cautious with interpretation of the data. Additional studies are needed to confirm our findings. However, critically ill patients with primary influenza A/H1N1 are challenging to prospectively identify and recruit. Thus, smaller studies of this nature are not uncommon within this field.

We also found that plasma IL-8 levels significantly correlated with MV-TF activity in influenza patients. Notably, there were no differences between influenza survivors and nonsurvivors with regards to demographics, illness-severity, shock, DIC, or the need for and duration of mechanical ventilation. Thus, we do not believe that our findings that MV-TF activity and IL-8 were associated with mortality are due simply to confounding bias. Moreover, our findings highlight that traditional risk factors do not correlate with influenzarelated mortality, as also described by others (2).

While the precise mechanism underlying our findings that MV-TF activity and IL-8 were significantly correlated and predicted influenza mortality are unknown, we hypothesize that convergent signaling pathways through monocytes, macrophages, or other cells may contribute to the increased MV-TF activity and IL-8 levels seen in our patients. Taken together, the findings of our study demonstrate, for the first time to our knowledge, that MVTF activity and IL-8 levels may have clinical utility as early biomarkers to identify influenza patients at increased risk of mortality.

\section{Conclusions}

This study demonstrates that MV-TF activity and IL-8 levels in patients with severe, primary influenza A/H1N1 infection are early markers associated with subsequent influenza-related mortality.

\section{Acknowledgments}

Financial Support: This work was supported by the NIH (HL092161, HL112311 to MTR, HL090785 to JAB \& HL119523 to NM) and the NIA (AG040631, AG048022 to MTR). 


\section{References}

1. Mulrennan S, Tempone SS, Ling IT, et al. Pandemic influenza (H1N1) 2009 pneumonia: CURB-65 score for predicting severity and nasopharyngeal sampling for diagnosis are unreliable. PloS one. 2010; 5(9):e12849. [PubMed: 20877727]

2. Bunce PE, High SM, Nadjafi M, et al. Pandemic H1N1 influenza infection and vascular thrombosis. Clin Infect Dis. 2011; 52(2):e14-e17. [PubMed: 21288835]

3. Rondina MT, Brewster B, Grissom CK, et al. In Vivo Platelet Activation in Critically Ill Patients With Primary 2009 Influenza A(H1N1). Chest. 2012; 141(6):1490-1495. [PubMed: 22383669]

4. Owens AP 3rd, Mackman N. Microparticles in hemostasis and thrombosis. Circ Res. 2011; 108(10): 1284-1297. [PubMed: 21566224]

5. Antoniak S, Mackman N. Multiple roles of the coagulation protease cascade during virus infection. Blood. 2014; 123(17):2605-2613. [PubMed: 24632711]

6. Boilard E, Pare G, Rousseau M, et al. Influenza virus H1N1 activates platelets through FcgammaRIIA signaling and thrombin generation. Blood. 2014

7. Khorana AA, Francis CW, Menzies KE, et al. Plasma tissue factor may be predictive of venous thromboembolism in pancreatic cancer. J Thromb Haemost. 2008; 6(11):1983-1985. [PubMed: 18795992]

8. de Jong MD, Simmons CP, Thanh TT, et al. Fatal outcome of human influenza A (H5N1) is associated with high viral load and hypercytokinemia. Nature medicine. 2006; 12(10):1203-1207.

9. Meziani F, Delabranche X, Asfar P, et al. Bench-to-bedside review: circulating microparticles--a new player in sepsis? Crit Care. 2010; 14(5):236. [PubMed: 21067540]

10. Morel O, Ohlmann P, Epailly E, et al. Endothelial cell activation contributes to the release of procoagulant microparticles during acute cardiac allograft rejection. The Journal of heart and lung transplantation : the official publication of the International Society for Heart Transplantation. 2008; 27(1):38-45.

11. Matsumoto H, Yamakawa K, Ogura H, et al. Enhanced expression of cell-specific surface antigens on endothelial microparticles in sepsis-induced disseminated intravascular coagulation. Shock. 2015; 43(5):443-449. [PubMed: 25608138] 


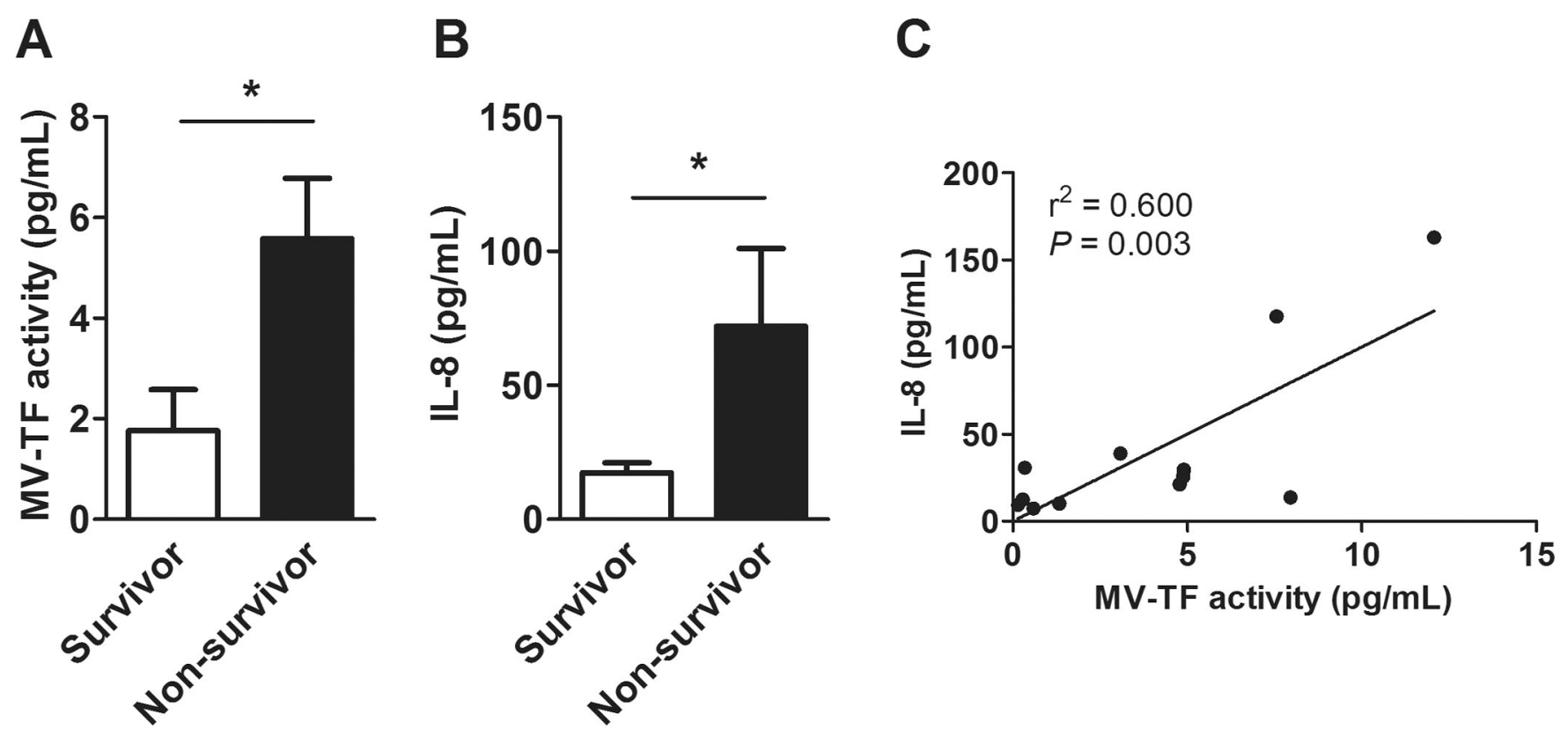

Figure 1. MV-TF activity and IL-8 as biomarkers are associated with mortality in patients with influenza $\mathrm{A} / \mathrm{H} 1 \mathrm{N1}$ infection

(A) Plasma levels of microvesicle tissue factor $(\mathrm{MV}-\mathrm{TF})$ activity in survivors (white, $\mathrm{n}=7$ ) and non-survivors (black, $\mathrm{n}=8$ ). (B) Plasma levels of interleukin 8 (IL-8) in survivors

(white, $n=7$ ) and non-survivors (black, $n=5$ ). (C) Correlation between MV-TF activity and IL-8 levels in H1N1 influenza-infected patients are shown $(\mathrm{n}=12$, each). Data (mean \pm SEM) were analyzed by Student's $t$-test (A and B) and linear regression analyses (C), and statistical significances are shown as $* P<0.05$. 


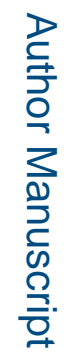

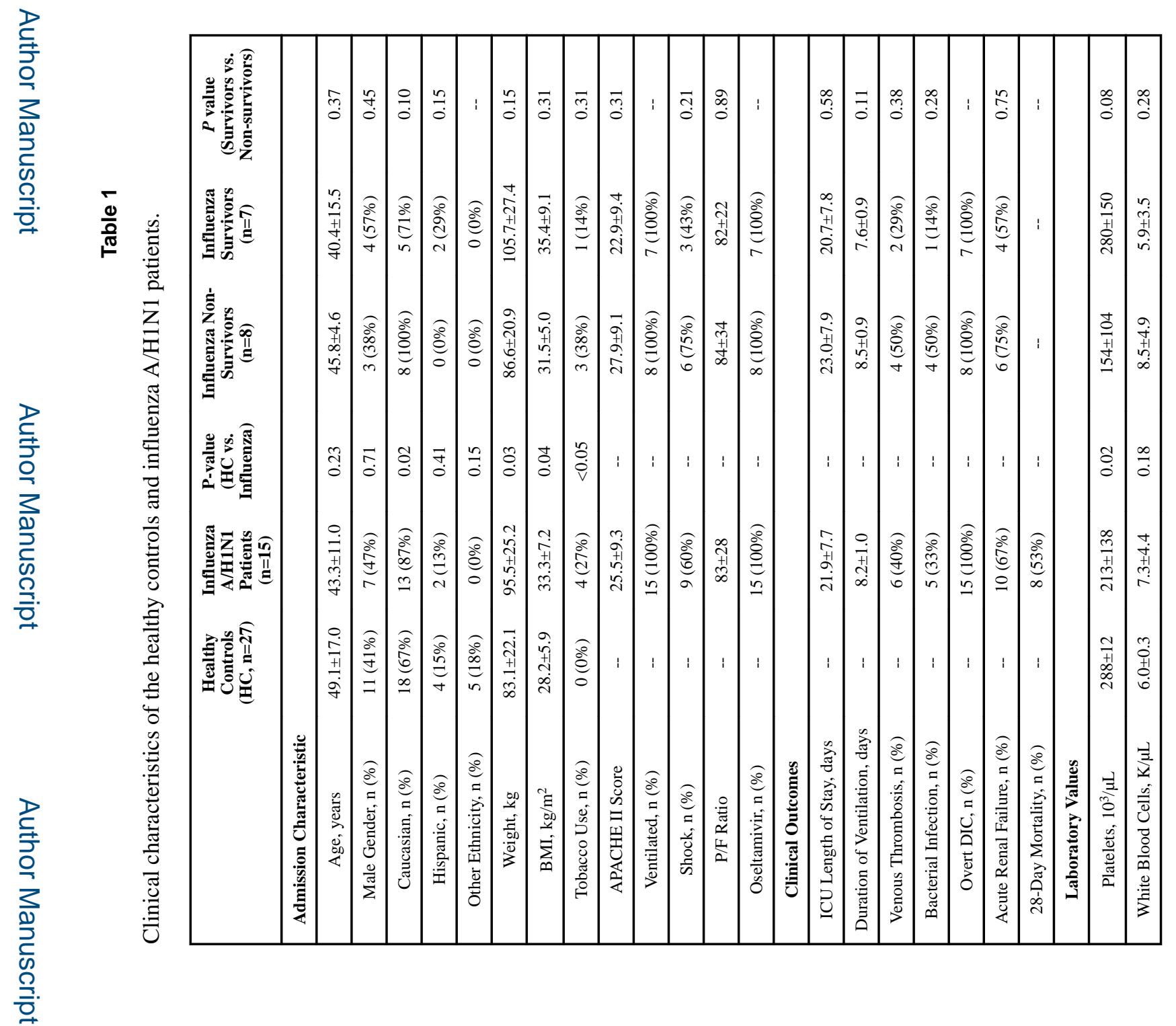

Crit Care Med. Author manuscript; available in PMC 2017 July 01. 


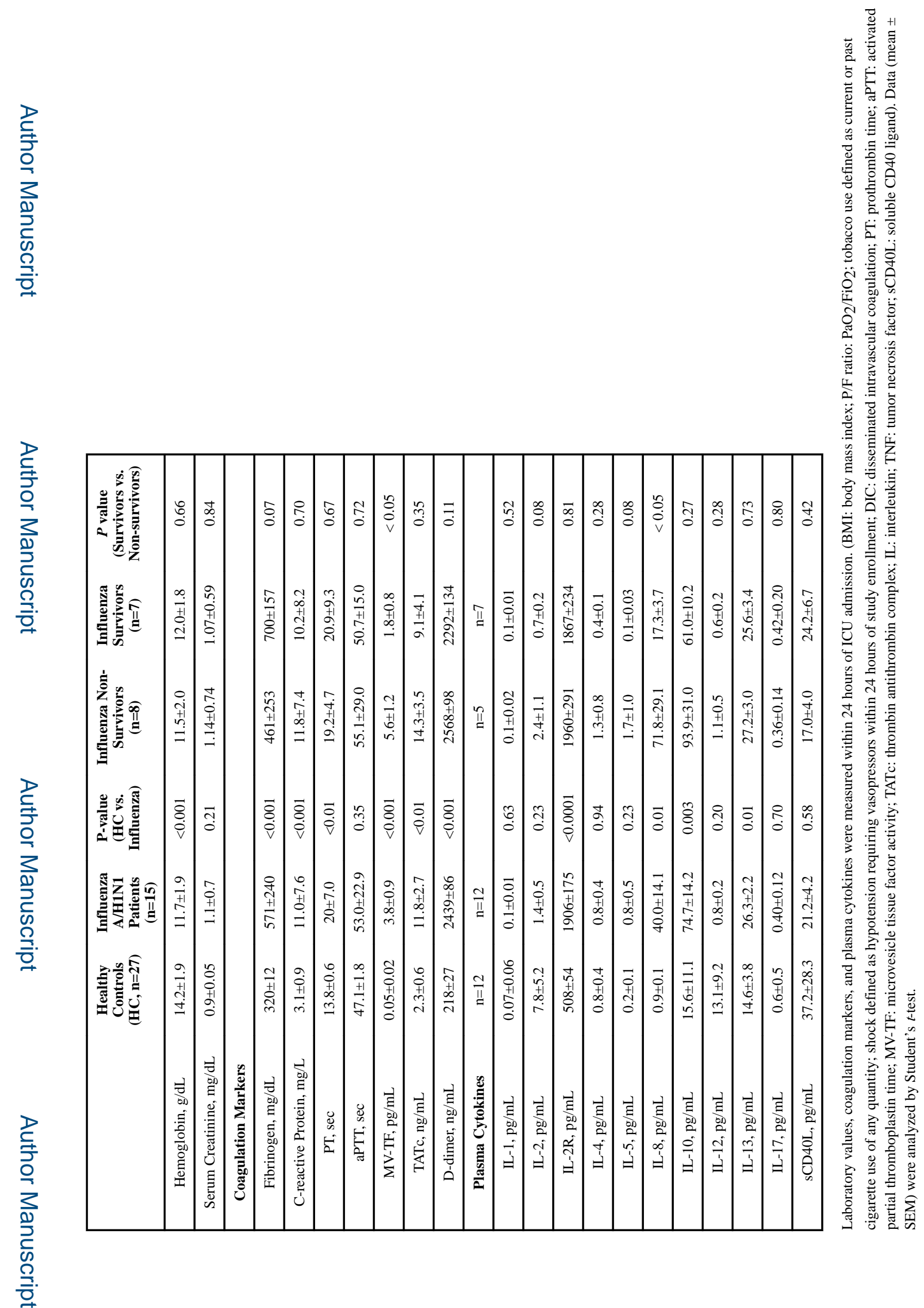

Crit Care Med. Author manuscript; available in PMC 2017 July 01. 